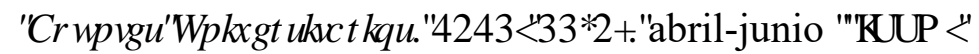

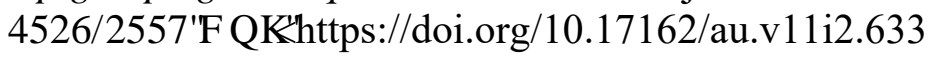

\title{
Ética y TIC: Algunas reflexiones sobre el rol de los educadores
}

\section{Ethics and ICT. Some reflections on the role of teachers}

\author{
Karla Bustos Canales ${ }^{1 \mathrm{a}}$ \\ Universidad de Concepción, Campus Los Ángeles, Chile ${ }^{1}$
}

(iD) ORCID ID: https://orcid.org/0000-0002-8792-74281

Recibido: 16 de octubre 2020

Aceptado: 10 de diciembre de 2020

\begin{abstract}
Resumen
El propósito de este trabajo es reflexionar sobre la implicancia de la Ética en las Tecnologías de la Información y de la Comunicación (TIC) en contextos de aprendizaje escolar. En primer lugar, se identifican algunos problemas relevantes en el uso de las herramientas tecnológicas, esto es, precisar aquellas dificultades que requieren de la supervisión y orientación de un educador. Luego, se enfatiza en la necesidad de actualizar los protocolos que regulan las conductas y los comportamientos de los usuarios en la red, especialmente, cuando participan comunidades educativas conformadas por niños y adolescentes. Finalmente, se entregan algunas orientaciones pertinentes a la redacción de protocolos que reglamenten las conductas del estudiantado en el espacio digital.
\end{abstract}

Palabras clave: TIC, ética, cultura digital, educación, aprendizaje escolar, tecnología.

\footnotetext{
Abstract

This article aims to reflect on the implications of the ethics in the use of the information and communications technology (ICT) in learning contexts at school. Firstly, some relevant issues concerning the use of these tools are identified, specifying those difficulties which require guidance and orientation from a teacher. Then, we emphasize the need to update the

${ }^{\mathrm{a} C}$ Correspondencia al autor:

E-mail: karlabustos2016@udec.cl
} 
protocols that regulate the behaviors of the internet users, especially, when they involve children and teenagers in educational communities. Finally, some proposals to guide future papers about this topic are given, in order to establish good behavior guidelines for the students in the digital era.

Keywords: ICT, ethic, digital culture, education, school learning, technology

\section{Introducción}

En los últimos años, las Tecnologías de la Información y de la Comunicación, TIC, han dado pasos importantes para facilitar y agilizar algunos procesos que, en épocas anteriores, habrían tardado y requerido esfuerzos mayores. Debido al desarrollo y la masificación de las herramientas digitales, resulta complejo precisar un aspecto de nuestras vidas que prescinda del uso de la tecnología.

El desarrollo tecnológico incide en la rutina de las personas, pues todos consideramos -al menos- un dispositivo electrónico en nuestros quehaceres cotidianos; ya sea para fines recreativos, propósitos laborales, comunicacionales, entre otros asuntos. La relevancia que alcanzó la tecnología en nuestro país, Chile, se comprueba luego de referir un estudio que publicó el Instituto Nacional de Estadística (Petersen, 2018), que precisa la existencia de 28 millones de dispositivos móviles operativos para una población que no supera los veinte millones de habitantes, es decir, cada residente tendría a su disposición 1.5 aparatos celulares.

La relevancia de las TIC y los beneficios que aportan estas herramientas digitales son indiscutibles, sin embargo, han surgido una serie de cuestionamientos que exigen precisar cuáles son los límites que definen el uso lícito de estas tecnologías. En este contexto, Montuschi (2005) propuso que un empleo legítimo de las herramientas digitales debe considerar tres aspectos fundamentales: información, privacidad y propiedad.

Aunque el estudio de Montuschi tiene algunos años de antigüedad, esta reflexión es pionera en su campo de estudio, pues revela una problemática que no se ha estudiado con la rigurosidad y sistematicidad que exige la investigación académica. El trabajo de esta investigadora precisó los efectos que ejercen las TIC en el mundo del trabajo y en las relaciones interpersonales, en pocas palabras, Montischi sugiere trazar límites claros entre: “el trabajo, el aprendizaje y el ocio" (p.16). 
Los contextos de aprendizaje, especialmente aquellos espacios que consideran el ámbito escolar, no son ajenos a estas dificultades, ya que, docentes y estudiantes coinciden al estimar que las herramientas tecnológicas son apoyos fundamentales en las labores educativas. A partir de la relevancia de las TIC en las comunidades de aprendizaje, surgen algunas problemáticas que nos interesa evaluar en este artículo académico, esto es, indagar y especificar, desde una perspectiva ética, cuáles son los parámetros que deben considerar las políticas educacionales al momento de precisar los límites que definen el correcto uso de las TIC, asunto que exige definir en qué situaciones se vulnera la integridad del alumnado, cómo estas contrariedades afectan la calidad de los aprendizajes, en qué medidas estas dificultades perjudican las relaciones humanas, entre otros asuntos.

La metodología de trabajo empleada en este artículo es de carácter hermenéutica. Consideramos que este procedimiento reflexivo es el más adecuado para el tema estudiado ya que, ofrece la posibilidad de interpretar y elaborar conclusiones cuyo impacto amplía el horizonte de los estudios educativos, además, este método de investigación beneficia la elaboración de reflexiones propias mediante la indagación de un corpus bibliográfico específico.

\section{La relevancia de las TIC en los contextos de aprendizaje escolar}

Como se adelantó las herramientas tecnológicas favorecen el desarrollo de las labores docentes y la consolidación de los aprendizajes de los estudiantes. El protagonismo de las TIC en el ámbito educativo es de tal envergadura que cualquier actividad pedagógica que no considere herramientas digitales es descalificada, tildada de anticuada e incapaz de satisfacer las necesidades y requerimientos del alumnado. Esta apreciación, un tanto personal, es compartida por Sánchez y Ponce (2007), quienes indican que las acciones educativas tradicionales, por sí mismas, no son suficientes para que los alumnos desarrollen las habilidades que aseguran su desenvolvimiento pleno en el mundo laboral.

En términos generales, el impacto de las TIC en el quehacer pedagógico es un asunto que no admite cuestionamientos. Basta con mencionar las restricciones y dificultades que impuso el Covid-19 a las comunidades educativas durante el periodo 2020 para evidenciar el protagonismo de las herramientas digitales en el ámbito educativo, pues, a fin de cuentas, fueron las plataformas tecnológicas las responsables de sostener el sistema educativo 
mediante la enseñanza a distancia. El protagonismo de las herramientas digitales en los procesos educativos es indiscutible, relevancia que conlleva una serie de dificultades vinculadas a su uso, especialmente, en lo que respecta a la implicancia de la ética en el campo tecnológico. Acerca de estas complejidades se discute en el siguiente apartado.

\section{La ética y su implicancia en las TIC}

Antes de reflexionar sobre en las complejidades que se suscitan, en torno a la ética y las TIC, es necesario definir ambos conceptos. Se entiende por ética aquella rama de la "filosofía que estudia las leyes de la licitud, o moralidad de los actos, y su fundamento" (Torres, 2014, p.9); en palabras sencillas, la ética es una rama de la filosofía cuyo objeto de estudio es la conducta de los hombres en sociedad. A su vez, siguiendo a Vivas (2018), las TIC son un "conjunto de recursos, herramientas, equipos, programas informáticos, aplicaciones, redes y medios, que permiten la compilación, procesamiento, almacenamiento, transmisión de información como: voz, datos, texto, vídeo e imágenes” (p. 241).

La relación que entabla la ética con las TIC es compleja, especialmente, si consideramos el papel que desempeñan las herramientas digitales en las comunidades de aprendizaje, ya que, estas tecnologías han desencadenado cambios significativos en las metodologías que conlleva la labor docente. A causa de esta preponderancia, las TIC han devenido objeto de estudio de diversas disciplinas, por ejemplo, de la Filosofía, la Sociología y la Antropología; además, estas herramientas tecnológicas han conformado su propio campo de saberes, denominado: “Ciencia, Tecnología y Sociedad” (Vivas, p.239).

A esta discusión epistemológica se incorpora un nuevo concepto: "tecno-ética". Este neologismo abre sendas reflexivas cuyo propósito es regular el correcto uso de las nuevas tecnologías (Vivas, p. 239). Respecto de los alcances de la "tecnoética", es preciso apuntar algunas investigaciones especializadas que delimitan los alcances teóricos de esta conceptualización, por ejemplo, Luppicini y Adell indican que: "la tecnoética es un campo de investigación interdisciplinar en el que se estudian los aspectos éticos y morales de la tecnología en la sociedad" (citado en Vivas p. 240).

Siguiendo esta línea de reflexión, es interesante singularizar algunos problemas que se derivan, a partir del mal uso de las herramientas tecnológicas, contrariedades que, desde una perspectiva ética, propician las siguientes dificultades: a) transgresión de los derechos de 
autor y de privacidad; b) suplantación de identidad; c) el ciberacoso; d) el sexting, es decir, el envío de contenidos eróticos entre dispositivos móviles (Molina et al., p.34); e) el cyberbullying, modalidad que implica el uso de las redes sociales y demás herramientas tecnológicas para agredir, discriminar, ridiculizar o hacer sentir mal a una víctima (Martínez, p.16); f) el grooming, conducta en la que un adulto establece vínculos socioemocional con un menor, fingiendo amistad, cariño, comprensión, ayuda, entre otros, para obtener abusar sexualmente (Molina et al., p. 34), etcétera.

Estas prácticas vulneran gravemente los derechos de las víctimas, por consiguiente, estas trasgresiones constituyen una preocupación relevante para los gobiernos y las autoridades educativas de todos nuestros países.

Esta arista de la discusión dialoga con la opinión de Vivas (2018), quien indica que las vulneraciones apuntadas, interfieren, de manera significativa, en la vida de los usuarios; especialmente, cuando involucran contextos de infancia y de adolescencia. Estas dificultades exigen la preocupación y el cuidado por parte de los adultos, ya que, se enmarcan en espacios de aprendizaje escolar. Por consiguiente, cuando se conjugan las áreas tecnológicas y educativas, la figura del docente asume un rol protagónico, pues el profesorado, junto con los padres y tutores, es responsables de prevenir cualquier instancia de vulneración que afecte a un menor. De la labor de los educadores, en cuanto al aprendizaje y la consolidación de comportamientos éticos del estudiantado en el espacio digital, se reflexiona en el siguiente apartado.

\section{La labor ética del educador en la era digital}

La capacitación docente es un fundamento en la gestión pedagógica, principalmente, cuando considera la adquisición de metodologías innovadoras en el proceso de enseñanzaaprendizaje. A causa de esta relevancia, muchos profesores se capacitan, año tras año, en la consolidación de una praxis docente que favorezca su labor educativa. Esta continua actualización permite suponer, mediante fundamentos sólidos, que el profesorado es consciente del beneficio que otorgan las nuevas tecnologías al desarrollo de las tareas pedagógicas, ya que, "los ambientes de aprendizaje que resultan más efectivos son los que mezclan enfoques tradicionales y nuevos para facilitar el aprendizaje de contenidos" (Sánchez y Ponce, 2007, p. 346). 
A estas contribuciones se añade la inmersión del alumnado en la "Era digital", pues la mayoría de nuestros estudiantes crecieron en ambientes altamente tecnologizados. A causa de estos antecedentes, al estudiantado le resulta "natural" acceder a una cantidad importante de información y de contenidos sin realizar esfuerzos significativos. Pareciera que han quedado en el pasado, o bien, cada vez son menos frecuentes, las largas horas de investigación bibliográfica en las hemerotecas de las escuelas y de las universidades.

Esta realidad ha compelido a los educadores a devenir sujetos competentes en el manejo de las herramientas digitales, docentes cuya praxis pedagógica debe considerar la evaluación permanente de metodologías y estrategias didácticas en la sala de clases.

Esta urgencia pedagógica explica por qué el profesorado, en muchas ocasiones, centra sus esfuerzos en la adquisición de herramientas digitales, descuidando la formación del alumnado en la consolidación de comportamientos éticos en el uso de herramientas tecnológicas. Así lo indican Viñals y Cuenca (2016), quienes sostienen que no es suficiente con manejar la tecnología, pues, para ser un docente competente en el área digital, es imprescindible que cada maestro determine sus propias necesidades formativas y de desarrollo personal (p. 104).

Por consiguiente, el educador, además de ser un facilitador de conocimientos, también debe asumir la responsabilidad de orientar a los estudiantes en el manejo, "correcto y lícito", de las prácticas en el espacio digital. En este sentido, todo profesor debe tutelar a sus alumnos en los procesos de búsqueda de información, análisis de datos, interpretación de contenidos, difusión de antecedentes personales, entre otras prácticas digitales. No obstante, las relevancias de estas tareas pedagógicas, Viñals y Cuenca subrayan que el docente, de ningún modo, debe asumir el rol de un "policía digital”, únicamente, el profesorado habrá de cautelar que las actividades que realizan sus estudiantes son legales y seguras (p. 110).

Si se piensa esta problemática, a partir de la realidad educativa que nos convoca, la chilena, es posible mencionar la iniciativa que formula el departamento de Ciencias de la Computación de la Universidad de Chile para establecer estándares TIC con el propósito de definir cuáles son los parámetros éticos que rigen una práctica pertinente en el uso de las herramientas tecnológicas, garantizando un empleo seguro y saludable de los recursos disponibles (Sánchez y Ponce, 2007). En pocas palabras, este protocolo evidencia la preocupación de las autoridades nacionales por delimitar las conductas éticas involucradas 
en el desarrollo saludable de las TIC. Una inquietud permanente en el profesorado, asunto pedagógico sobre el que se suele hablar mucho, pero se investigar bastante poco.

Los antecedentes que expuestos en este artículo, hasta esta parte, evidencian la necesidad de capacitar al cuerpo docente en el uso ético de las herramientas tecnológicas. Esta actualización de conocimientos permitirá que el profesorado ejerza labores de orientación, en cuanto a la utilización responsable y segura de los recursos tecnológicos, ya sea, en el ámbito escolar, o bien, en contextos extraprogramáticos. En este sentido, es oportuno que todas las comunidades educativas trabajen, colaborativamente, en la adquisición de competencias digitales para garantizar la consolidación de conocimientos técnicos, el desarrollo de habilidades y, especialmente, la integración de comportamientos éticos que favorezcan el autocuidado y el respeto por todos los usuarios que navegan en la red digital.

La comunidad educativa europea muestra ventajas considerables en el tratamiento de estas materias respecto del trabajo que se realiza a nivel nacional y en toda Latinoamérica. Las autoridades del viejo continente canalizaron estas preocupaciones en la redacción del protocolo A Framework for Developing and Understanding Digital Competence in Europe (2013). Esta iniciativa propuso que todo usuario digital, sea profesor o alumno, debe adoptar cinco actitudes fundamentales al momento de ingresar al espacio digital (Viñals y Cuenca, p. 108):

1. Tratamiento de la información: esto implica identificar, localizar, recuperar, almacenar, organizar y analizar la información digital.

2. Comunicación de la información recopilada: este punto supone compartir datos, a través de herramientas en línea, conectar y colaborar con otros usuarios.

3. Creación de contenido: edición y producción de contenidos (textos, imágenes, videos, etc.).

4. Seguridad: incentivo de la protección de antecedentes y datos personales, mediante una conducta segura y sostenida en el tiempo.

5. Resolución de problemas: identificar necesidades y recursos digitales, tomando decisiones respecto de la elección de una herramienta digital apropiada al fin esperado 
A partir del carácter universal de las TIC, valoramos el protocolo europeo, a modo de una referencia capaz de sintetizar las conductas apropiadas en el empleo de las herramientas digitales. En otras palabras, no basta con capacitar a los docentes en el conocimiento específico de un software o una plataforma web determinada, también es necesario asegurar la consolidación de los aprendizajes del cuerpo docente respecto de las habilidades mencionadas en el punto cuatro, esto es, cautelar la adquisición de competencias vinculadas a la promoción de comportamientos éticos en el espacio digital.

A partir de lo expuesto en este artículo, se evidencia necesidad de definir protocolos que regulen el comportamiento y la conducta de los usuarios en la red digital, particularmente, si se considera la inminente masificación de la tecnología 5G, es decir, el "Internet de las cosas". Este adelanto proporcionará acceso ilimitado e instantáneo a la información y otorgará la posibilidad de compartir datos en cualquier parte, a toda hora, con cualquier persona o cosa (González y Salamanca, 2016).

En definitiva, las TIC han llegado para quedarse y, conforme con la evolución de las herramientas digitales, también lo hará la vida de los usuarios. Esta situación obliga a pensar y a reformular los fundamentos que sostendrán la nueva era digital que se avecina, una reflexión en la que los educadores son interlocutores -permitentes y relevantes- que no deben ausentarse de este debate.

\section{Conclusión}

Las conclusiones que propone este artículo son de carácter provisorias, ya que, resultaría improcedente sugerir categorizaciones rígidas y estables para un asunto cuyas formulaciones se encuentran en plena discusión. En este sentido, es necesario reconocer la insuficiencia de las autoridades chilenas en la redacción y divulgación de protocolos que regulen el comportamiento de los estudiantes en el espacio digital. La carencia de reglamentos se extiende a buena parte de los países latinoamericanos, cuyas políticas educativas tampoco han considerado la elaboración de normativas éticas que distingan las prácticas lícitas en el espacio digital. Estas dificultades son aún más relevantes cuando involucran contextos de aprendizaje escolar, pues, la ausencia reglamentos que normalicen el comportamiento de los usuarios propicia instancias de vulneración del estudiantado. 
Las proyecciones que se pueden trazar, a partir de las aristas de reflexión que ofrece este artículo son variadas. En primer orden, enfatizar en la necesidad de educar a padres y apoderados en el uso ético de las TIC para que contribuyan en la formación de sus pupilos, especialmente, en aquellas en instancias que se desarrollan fuera del horario escolar. En segundo término, investigar el impacto en la consolidación de los aprendizajes del alumnado cuando median herramientas tecnológicas, es decir, indagar en qué medida las TIC favorecen los aprendizajes significativos en aquellos estudiantes que mantienen contacto permanente con sus profesores y compañeros mediante plataformas electrónicas. Finalmente, sería prudente evaluar cómo el contexto pandémico ha influido en el modo en que el estudiantado se relaciona con las herramientas digitales, pues el confinamiento sanitario y la educación a distancia han obligado a los alumnos a forjar una relación especial con las TIC, vínculo que, algunos meses atrás, antes del Covid-19, solía ser limitado y adscrito a situaciones específicas.

\section{Referencias}

González, J. y Salamanca, O. (2016). El camino hacia la tecnología 5G. Télématique, 15(1), 27-47. Recuperado de https://www.redalyc.org/pdf/784/78445977002.pdf

Martínez, J. (2017). Bullying, Sexting y Grooming. ¡Cómo los puedes prevenir y combatir! Bogotá: San Pablo.

Molina, J. y Vecina, P. (2015). Bullying, ciberbullying y sexting. ¿Cómo actuar ante una situación de acoso? Madrid: Ediciones Pirámide.

Montuschi, L. (2005). Aspectos éticos de las tecnologías de la información y de la comunicación: La ética de la computación, internet y la world wide web. Econstor, 298, 1-28. Recuperado de https://ideas.repec.org/p/cem/doctra/298.html

Petersen, V. (10 de febrero de 2018). Celulares en Chile se acercan a los 27 millones. La Tercera. Recuperado de http:// https://www.latercera.com/entretencion/noticia/celulares-chile-se-acercan-los-27millones/63290/

Sánchez, J. y Ponce, A. (2007). Estándares TIC para profesores chilenos. VII Congreso Iberoamericano de Información Educativa. Mar del Plata: Cepal, 345-354. 
Recuperado

de:

http://www.ufrgs.br/niee/eventos/RIBIE/2004/comunicacao/com345-354.pdf

Torres, Z. (2014). Introducción a la ética. México: Grupo Editorial Patria.

Viñals, A. y Cuenca, J. (2016). El rol del profesorado en la era digital. Revista Interuniversitaria de Formación del Profesorado, 30(2), 103-114. Recuperado de: https://www.redalyc.org/pdf/274/27447325008.pdf

Vivas, W. (2018). Uso seguro y responsable de las TIC: una aproximación desde la tecnoética. Ciencia, Docencia y Tecnología, 29(57), 235-255. Recuperado de: https://www.redalyc.org/jatsRepo/145/14560144009/html/index.html 